\title{
The potential threat of multisystem inflammatory syndrome in children during the COVID-19 pandemic
}

\author{
Hussin Rothan ${ }^{1}$ and Siddappa Byrareddy ${ }^{2}$ \\ ${ }^{1}$ Georgia State University \\ ${ }^{2}$ University of Nebraska Medical Center
}

June 15, 2020

\begin{abstract}
Multisystem inflammatory syndrome in children (MIS-C) during the COVID-19 pandemic raised a global alert from the Centers for Disease Control and Prevention's Health Alert Network. The main manifestations of MIS-C in the setting of a severe inflammatory state include fever, diarrhea, shock, and variable presence of rash, conjunctivitis, extremity edema, and mucous membrane changes, and in some cases it progressed to multi-organ failure. The low percentage of children with asymptomatic cases compared with mild illness and moderate illness could be correlated with the rare cases of MIS-C. One potential explanation for the progression to severe MIS-C disease despite the presence of readily detectable anti-SARS-CoV-2 antibodies could be due to potential role of antibody-dependent enhancement (ADE). We reason that the incidence of the ADE phenomenon whereby the pathogen-specific antibodies can promote pathology should be considered in vaccine development against SARS-COV-2.
\end{abstract}

\section{Introduction}

In April 2020, clinical reports documented the occurrence of a multisystem inflammatory syndrome in children (MIS-C) during the COVID-19 pandemic in the United States, United Kingdom, Italy, and France. On May 14, 2020, the Centers for Disease Control and Prevention's Health Alert Network issued a global alert for MIS-C after identifying a possible link between this critical illness in children and infection from the novel coronavirus SARS-COV-2, the virus that causes the COVID-19 disease[1]. The main manifestations of MIS-C in the setting of a severe inflammatory state include fever, diarrhea, shock, and variable presence of rash, conjunctivitis, extremity edema, and mucous membrane changes, and in some cases this can lead to the development of multi-organ failure[2]. The multi-organ failure in MIS-C is manifest by neurologic involvement, hyperferritinemia, and cardiogenic or vasoplegic shock [3]. Children with severe MIS-C cases who have either previous exposure to SARS-CoV-2 or tested positive for SARS-CoV-2 were admitted to the intensive care units for shock or acute heart dysfunction [4].

The MIS-C features share some aspects of the Kawasaki syndrome in terms of multisystem inflammation and high levels of inflammatory biomarkers. However, whereas MIS-C has been reported in individuals who are upto 21 years of age with a higher rate of cardiac involvement [3], patients with Kawasaki syndrome has been shown to occur predominantly in infants and children under 5 years old [4]. The etiology of Kawasaki disease remains unknown, with evidence suggesting that infectious agents could trigger the initiation of this disease. Multiple cases of previously healthy children who developed MIS-C syndrome were shown to be associated with a clinical or subclinical SARS-COV-2 infection. At present, it is unclear whether the SARS-COV-2 infection could ignite the inflammation cascade that causes MIS-C illness.

Whereas it was previously thought that SARS-COV-2 infection shows mild to asymptomatic disease in children and those that become infected are not susceptible to pneumonia secondary to COVID-19 infection, 
this view has significantly changed following the documentation of MIS-C in select cases of pediatric SARSCoV-2 infection[5]. These findings has led to the conclusion that clearly more detailed studies are required to more precisely define the molecular aspects of the triggering factors of MIS-C during the COVID-19 pandemic.

\section{The low susceptibility of children to COVID-19 illness}

Adults with severe COVID-19 suffer from deadly pneumonia and insufficient supply of oxygen throughout the body, while children show mild to asymptomatic COVID-19 disease with fewer death cases. A study early in the COVID-19 pandemic showed that the percentage of infected children with SARS-COV-2 was as low as $0.9 \%$ for $0-10$ years, and $1.2 \%$ for $10-19$ years old [6]. Further analysis of the SARS-CoV-2 infected children showed that whereas there was a $4 \%$ incidence of children with asymptomatic cases, those with mild illness comprised 51\%, and those with moderate illness $39 \%$ of the total cases [7]. The molecular bases of the differences in COVID-19 pathogenesis between children and adults have yet to be fully understood.

The expression levels of SARS-COV-2 cellular receptors and co-receptors in the children and adults could have an impact on virus infectivity and disease severity. Angiotensin-converting enzyme-2 (ACE2) represents the primary SARS-COV-2 receptor for viral entry and it is co-expressed with a cluster of the transmembrane protease TMPRSS2. The role of TMPRSS2 is cleaving the S protein of SARS-COV-1 and SARS-COV-2 into two fragments $\mathrm{S} 1$, which is essential for virus attachment, and $\mathrm{S} 2$, for virus fusion into the target cells[8]. Attachment of the receptor-binding domain of the virus spikes to the ACE2 initiates SARS-COV entry into target cells $[9,10]$. As such, lung epithelial cells represent the coronaviruses' primary target because of the co-expression of the ACE2 receptor with TMPRSS2 protein[11].

Children show lower ACE2 expression in the lungs than adults, which could contribute to the observed differences in disease pathogenesis across different age groups [12]. It has been reported that SARS infection shows slight, non-specific, and cold-like symptoms in children younger than 12 years old that are less pronounced than it is in adolescents [13]. The expression levels of the TMPRSS2 protein is regulated by the levels of androgen and androgen receptors[14]. It is important to note that children younger than 12 years old have a lower level of androgen and androgen receptors than are present in adolescents and adult men. The age-based difference in the expression levels of ACE2 and TMPRSS2 could play a potential role in the severity of COVID-19 pathogenesis.

\section{The possible triggering of MIS-C via antibody-dependent enhancement}

A number of reports documented the finding that most of the individuals presenting with MIS-C have significant levels of SARS-COV-2 antibodies in their sera but they are negative for SARS-COV-2 by RTPCR. We reasoned that to a potential role of the antibody-dependent enhancement (ADE) that is well described in dengue and Zika virus infections[15]. ADE could trigger the MIS-C syndromes whereby the pathogen-specific antibodies can promote pathology.

It has been observed that the severe disease caused by SARS-CoV-1 infection is associated with the peak of neutralizing antibody response, suggesting that antibody responses that potentially contain ADE antibodies may also be related to disease outcome in SARS-CoV-1 infection [16]. The spike protein of SARS-CoV-2 contains various epitopes that could induce neutralizing and non-neutralizing antibody production. The neutralizing antibodies afford a protective effect against virus entry into the host cells. On the other hand, the antibodies generated against the non-neutralizing epitopes could enhance virus entry leading to severe disease outcomes.

We hypothesize that the initial exposure of children to the SARS-COV-2 induces both neutralizing and nonneutralizing antibodies production by immune cells. However, over time, it is possible that those children with predominantly virus neutralizing antibodies progress to asymptomatic COVID-19 illness. However, a select number of those that shift to producing predominantly non-neutralizing antibodies progress to severe disease due to ADE. This is exemplified by the finding that at low dilutions anti-sera against SARS-CoV neutralized SARS-CoV infection, while highly diluted anti-sera significantly increased SARS-CoV infection and induced 
higher levels of apoptosis [17]. Thus, the low levels of neutralizing antibodies could be insufficient to inhibit virus entry, while the low levels of non-neutralizing antibodies could enhance virus entry and worsen the disease outcome. It is important to note the delay between the recognized beginning of the SARS-COV-2 pandemic in the population and the recent emergence of MIS-C illness in children. It is possible that COVID19 induced pathology changed since the beginning of the pandemic, after the virus began circulating within the general population due to varied levels of generational immunity background of hosts. The severe or mild and symptomatic or asymptomatic infections produce a different ratio of neutralizing and non-neutralizing antibodies that will affect the pathology of the second infection with SARS-COV-2 (Figure 1).

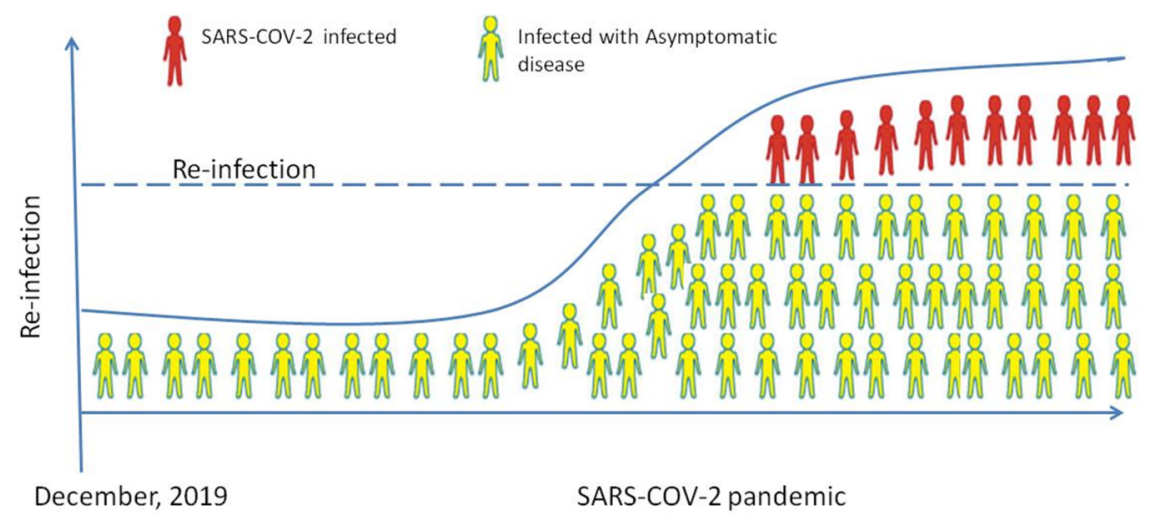

Figure 1: The hypothetical scheme shows that the susceptibility of children to re-infection with SARS-COV2 via ACE2-independent pathway will increase when the accumulated number of children with asymptomatic disease is increasing in the population.

\section{Microphages activation via antibody-dependent enhancement}

A growing body of evidence suggests the host's innate immune response to SARS-COV-2 infection triggers the inflammation cascade that causes severe tissue damage[18]. In select cases COVID-19 is characterized by a cytokine storm resembling that of macrophage activation seen in viral-induced lymphohistiocytosis and haemophagocytosis[19]. SARS-CoV-2 infects macrophages in the lung and other tissues leading to a surge in the production of pro-inflammatory cytokines.

The ACE2-independent pathway of virus entry depends on the expression of Fc receptors on the cellular membrane of the immune cells. Binding of virus-antibody complex to Fc receptors induces cellular endocytosis[17] (Figure 2). The existing SARS- CoV-2 specific antibodies in MIS-C patients may thus promote viral entry into immune cells resulting in immune cell activation and subsequent acute inflammation. The macrophages are activated when the endosomal Toll-like receptors TLR3, TLR7, and TLR8 start sensing viral RNA and induce a downstream cascade of pro-inflammatory cytokines such as TNF and IL-6[17]. The elevated levels of TNF are the leading cause of septic shock, and multi-organ failure may result in myocardial damage and circulatory failure observed in some COVID-9 patients [20]. The local inflammation and accumulation of pathological macrophage populations in the tissues could be among the leading causes of MIS-C syndrome. Further investigation is required to illustrate the role of macrophage populations in MIS-C syndrome. 


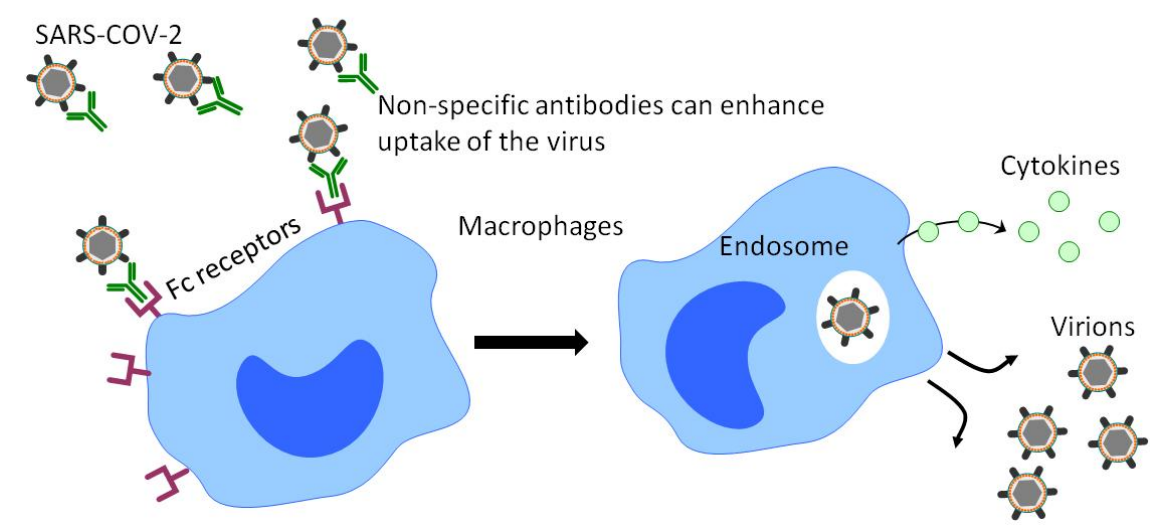

Figure 2: The ACE2-independent pathway of virus entry depends on the expression of Fc receptors on the cellular membrane of the immune cells. Binding of virus-antibody complex to Fc receptors induces cellular endocytosis resulting in immune cell activation and subsequent acute inflammation.

In conclusion, a few months after the COVID-19 pandemic, a significant frequency of MIS-C cases linked to SRAS-COV-2 infection were reported in different countries. There is an apparent correlation between the percentage of asymptomatic SRAS-COV-2 infection in children and the incidence of MIS-C. The absence of SARS-COV-2 RNA in seropositive individuals suggests the possible role of SARS-COV-2 non-specific antibodies in the development of MIS-C disease via ADE. As such, the phenomenon of ADE should be considered in vaccine development against SARS-COV-2.

Funding: This work is partially supported by National Institute of

Allergy and Infectious Diseases Grant R01 AI129745, National Institute of Mental Health Grant P30MH062261 and Frances E. Lageschulte and Evelyn B. Weese New Frontiers in Medical Research Fund to SNB.

\section{References}

1. https://emergency.cdc.gov/han/2020/han00432.asp.

2. Chiotos K, Bassiri H, Behrens EM, Blatz AM, Chang J, et al. (2020) Multisystem Inflammatory Syndrome in Children during the COVID-19 pandemic: a case series. J Pediatric Infect Dis Soc.

3. Hennon TR, Penque, M. D., Abdul-Aziz, R., Alibrahim, O. S., McGreevy, M. B., Prout, A. J., Schaefer, B. A., Ambrusko, S. J., Pastore, J. V., Turkovich, S. J., Gomez-Duarte, O. G., \& Hicar, M. D. (2020). COVID-19 associated Multisystem Inflammatory Syndrome in Children (MIS-C) guidelines; a Western New York approach. Progress in Pediatric Cardiology, 101232. Advance online publication. https://doi.org/10.1016/j.ppedcard.2020.101232.

4. Belhadjer Z, Meot M, Bajolle F, Khraiche D, Legendre A, et al. (2020) Acute heart failure in multisystem inflammatory syndrome in children (MIS-C) in the context of global SARS-CoV-2 pandemic. Circulation.

5. Wang D, Hu B, Hu C, Zhu F, Liu X, et al. (2020) Clinical Characteristics of 138 Hospitalized Patients With 2019 Novel Coronavirus-Infected Pneumonia in Wuhan, China. JAMA.

6. Novel Coronavirus Pneumonia Emergency Response Epidemiology T (2020) [The epidemiological characteristics of an outbreak of 2019 novel coronavirus diseases (COVID-19) in China]. Zhonghua Liu Xing Bing Xue Za Zhi 41: 145-151. 
7. Dong Y, Mo X, Hu Y, Qi X, Jiang F, et al. (2020) Epidemiological Characteristics of 2143 Pediatric Patients With 2019 Coronavirus Disease in China. Pediatrics.

8. Glowacka I, Bertram S, Muller MA, Allen P, Soilleux E, et al. (2011) Evidence that TMPRSS2 activates the severe acute respiratory syndrome coronavirus spike protein for membrane fusion and reduces viral control by the humoral immune response. J Virol 85: 4122-4134.

9. Wan Y, Shang J, Graham R, Baric RS, Li F (2020) Receptor recognition by novel coronavirus from Wuhan: An analysis based on decade-long structural studies of SARS. J Virol.

10. Jaimes JA, Millet JK, Stout AE, Andre NM, Whittaker GR (2020) A Tale of Two Viruses: The Distinct Spike Glycoproteins of Feline Coronaviruses. Viruses 12.

11. Jacquinet E, Rao NV, Rao GV, Hoidal JR (2000) Cloning, genomic organization, chromosomal assignment and expression of a novel mosaic serine proteinase: epitheliasin. FEBS Lett 468: 93-100.

12. Schouten LR, van Kaam AH, Kohse F, Veltkamp F, Bos LD, et al. (2019) Age-dependent differences in pulmonary host responses in ARDS: a prospective observational cohort study. Ann Intensive Care 9: 55.

13. Denison MR (2004) Severe acute respiratory syndrome coronavirus pathogenesis, disease and vaccines: an update. Pediatr Infect Dis J 23: S207-214.

14. Yu J, Yu J, Mani RS, Cao Q, Brenner CJ, et al. (2010) An integrated network of androgen receptor, polycomb, and TMPRSS2-ERG gene fusions in prostate cancer progression. Cancer Cell 17: 443-454.

15. Rothan HA, Bidokhti MRM, Byrareddy SN (2018) Current concerns and perspectives on Zika virus co-infection with arboviruses and HIV. J Autoimmun 89: 11-20.

16. Ho MS, Chen WJ, Chen HY, Lin SF, Wang MC, et al. (2005) Neutralizing antibody response and SARS severity. Emerg Infect Dis 11: 1730-1737.

17. Wang SF, Tseng SP, Yen CH, Yang JY, Tsao CH, et al. (2014) Antibody-dependent SARS coronavirus infection is mediated by antibodies against spike proteins. Biochem Biophys Res Commun 451: 208-214.

18. Rothan HA, Byrareddy SN (2020) The epidemiology and pathogenesis of coronavirus disease (COVID19) outbreak. J Autoimmun 109: 102433.

19. D'Antiga L (2020) Coronaviruses and Immunosuppressed Patients: The Facts During the Third Epidemic. Liver Transpl 26: 832-834.

20. Ruan Q, Yang K, Wang W, Jiang L, Song J (2020) Clinical predictors of mortality due to COVID-19 based on an analysis of data of 150 patients from Wuhan, China. Intensive Care Med 46: 846-848. 